\title{
Cytokine Degradation Process
}

National Cancer Institute

\section{Source}

National Cancer Institute. Cytokine Degradation Process. NCI Thesaurus. Code C40678.

Cytokine Degradation consists of conjug ation, transport, oxidation, and/or proteolysis of a class of soluble non-antibody paracrine or autocrine signaling glycoproteins (cytokines) secreted by immune cells that regulate immune responses through high affinity binding with specific receptors. Cytokines act as intercellular mediators regulating immune and inflammatory responses. Activation enhances cell proliferation and differentiation, secretion of other biologically active molecules, and responses to immune and inflammatory stimuli. 